\title{
Anal Rhabdomyosarcoma
}

National Cancer Institute

\section{Source}

National Cancer Institute. Anal Rhabdomyosarcoma. NCI Thesaurus. Code C5610.

A malignant mesenchymal tumor with skeletal muscle differentiation affecting the anus. 